\title{
On Local Existence and Blow-Up of Solutions for Nonlinear Wave Equations of Higher-Order Kirchhoff Type with Strong Dissipation
}

\author{
Guoguang Lin, Yunlong Gao, Yuting Sun \\ Department of Mathematics, Yunnan University, Kunming, China \\ Email: gglin@ynu.edu.cn,gyl0813101x@163.com, syt19911006@163.com
}

How to cite this paper: Lin, G.G., Gao, Y.L. and Sun, Y.T. (2017) On Local Existence and Blow-Up of Solutions for Nonlinear Wave Equations of Higher-Order Kirchhoff Type with Strong Dissipation. International Journal of Modern Nonlinear Theory and Application, 6, 11-25. https://doi.org/10.4236/ijmnta.2017.61002

Received: January 5, 2017

Accepted: February 10, 2017

Published: February 13, 2017

Copyright (c) 2017 by authors and Scientific Research Publishing Inc. This work is licensed under the Creative Commons Attribution International License (CC BY 4.0).

http://creativecommons.org/licenses/by/4.0/

\begin{abstract}
In this paper, we study on the initial-boundary value problem for nonlinear wave equations of higher-order Kirchhoff type with Strong Dissipation: $u_{t t}+(-\Delta)^{m} u_{t}+\left(a+b\left\|D^{m} u\right\|^{2 q}\right)(-\Delta)^{m} u=|u|^{p} u$. At first, we prove the existence and uniqueness of the local solution by the Banach contraction mapping principle. Then, by "Concavity" method we establish three blow-up results for certain solutions in the case 1): $E(0)<0$, in the case 2): $E(0)=0$ and in the case 3): $E(0)>0$. At last, we consider that the estimation of the upper bounds of the blow-up time $T^{*-}$ is given for deferent initial energy.

\section{Keywords}

Nonlinear Higher-Order Kirchhoff Type Equation, Strong Damping, Local Solutions, Blow-Up, Initial Energy
\end{abstract}

\section{Introduction}

In this paper, we are concerned with local existence and blow-up of the solution for nonlinear wave equations of Higher-order Kirchhoff type with strong dissipation:

$$
\begin{aligned}
& u_{t t}+(-\Delta)^{m} u_{t}+\left(a+b\left\|D^{m} u\right\|^{2 q}\right)(-\Delta)^{m} u=|u|^{p} u,(x, t) \in \Omega \times[0,+\infty), \\
& u(x, t)=0, \frac{\partial^{i} u}{\partial v^{i}}=0, i=1,2, \cdots, m-1, x \in \partial \Omega, t \in(0,+\infty), \\
& u(x, 0)=u_{0}(x), u_{t}(x, 0)=u_{1}(x), x \in \Omega,
\end{aligned}
$$

where $\Omega$ is a bounded domain in $R^{n}$ with the smooth boundary $\partial \Omega$ and $v$ 
is the unit outward normal on $\partial \Omega$. Moreover, $m>1$ is an integer constant, and $q, p, a$ and $b$ are some constants such that $q \geq 1, p \geq 0, a \geq 0$, $b \geq 0$ and $a+b>0$. We call Equation (1.1) a non-degenerate equation when $a>0$ and $b>0$, and a degenerate one when $a=0$ and $b>0$. In the case of $a>0$ and $b=0$, Equation (1.1) is usual semilinear wave equations.

It is known that Kirchhoff [1] first investigated the following nonlinear vibration of an elastic string for $\delta=f=0$ :

$$
\rho h \frac{\partial^{2} u}{\partial t^{2}}+\delta \frac{\partial u}{\partial t}=\left\{p_{0}+\frac{E h}{2 L} \int_{0}^{L}\left(\frac{\partial u}{\partial x}\right)^{2} \mathrm{~d} x\right\} \frac{\partial^{2} u}{\partial x^{2}}+f ; \quad 0 \leq x \leq L, t \geq 0,
$$

where $u=u(x, t)$ is the lateral displacement at the space coordinate $x$ and the time $t ; \rho$ : the mass density; $h$ : the cross-section area; $L$ : the length; $E$ : the Young modulus; $p 0$ : the initial axial tension; $\delta$ : the resistance modulus; and $f$ : the external force.

When $a=1, b=0, m=1$, the Equation (1.1) becomes a nonlinear wave equation:

$$
\begin{aligned}
& u_{t t}-\Delta u-\Delta u_{t}=|u|^{p} u, \quad(x, t) \in \Omega \times[0,+\infty), \\
& u(x, 0)=u_{0}(x), u_{t}(x, 0)=u_{1}(x), \quad x \in \Omega, \\
& u(x, t)=0, \quad(x, t) \in \partial \Omega \times[0,+\infty) .
\end{aligned}
$$

It has been extensively studied and several results concerning existence and blowing-up have been established [2] [3] [4].

When $a>0, b \geq 0, m=1$, the Equation (1.1) becomes the following Kirchhoff equation with Lipschitz type continuous coefficient and strong damping:

$$
\begin{aligned}
& u_{t t}-M\left(\|\nabla u\|^{2}\right) \Delta u-\omega \Delta u_{t}=|u|^{p} u, \\
& u(x, 0)=u_{0}(x), u_{t}(x, 0)=u_{1}(x), \quad x \in \Omega, \\
& u(x, t)=0, \quad(x, t) \in \partial \Omega \times[0, T],
\end{aligned}
$$

where $\Omega \in R^{N}, N \geq 1$ is a bounded domain with a smooth boundary $\partial \Omega \cdot p>$ 2 and $M(s)=m_{0}+b s^{\gamma}$ is a positive local Lipschitz function. Here, $m_{0}>0$, $b \geq 0, \gamma \geq 1, s \geq 0$. It has been studied and several results concerning existence and blowing-up have been established [5].

When $m=1$, the Equation (1.1) becomes the following Kirchhoff equation:

$$
\begin{aligned}
& u_{t t}-\left(a+b\left\|D^{m} u\right\|^{2 q}\right) \Delta u-\Delta u_{t}=|u|^{p} u, \quad(x, t) \in \Omega \times[0,+\infty), \\
& u(x, 0)=u_{0}(x), \quad u_{t}(x, 0)=u_{1}(x), x \in \Omega, \\
& u(x, t)=0, \quad(x, t) \in \partial \Omega \times[0,+\infty),
\end{aligned}
$$

where $\Omega$ is a bounded domain in $R^{n}$ with the smooth boundary $\partial \Omega$ and $v$ is the unit outward normal on $\partial \Omega$. Moreover, $q, p, a$ and $b$ are some constants such that $q \geq 1, p \geq 0, a \geq 0, b \geq 0$ and $a+b>0$. It has been studied and several results concerning existence and blowing-up have been established [6]. 
When $m=1$, reference [7] has considered global existence and decay estimates for nonlinear Kirchhoff-type equation:

$$
\begin{aligned}
& u_{t t}-\varphi\left(\|\nabla u\|^{2}\right) \Delta u-a \Delta u_{t}=b|u|^{\beta-2} u, \quad(x, t) \in \Omega \times(0,+\infty), \\
& u(x, t)=0, \quad(x, t) \in \Gamma_{1} \times(0,+\infty) \\
& \varphi\left(\|\nabla u\|^{2}\right) \frac{\partial u}{\partial v}+a \frac{\partial u_{t}}{\partial v}=g\left(u_{t}\right), \quad(x, t) \in \Gamma_{0} \times(0,+\infty), \\
& u(x, 0)=u_{0}(x), \quad u_{t}(x, 0)=u_{1}(x), \quad x \in \Omega
\end{aligned}
$$

where $\Omega$ is a bounded domain of $R^{n}(n \geq 1)$ with smooth boundary $\Gamma:=\partial \Omega$ such that $\Gamma=\Gamma_{0} \cup \Gamma_{1}$ and $\Gamma_{0}, \Gamma_{1}$ have positive measures, and $v$ is the unit outward normal on $\partial \Omega$, and $\frac{\partial}{\partial v}$ is the outward normal derivative on $\partial \Omega$.

In this paper we shall deal with local existence and blow-up of solutions for nonlinear wave equations of higher-order Kirchhoff type with strong dissipation. The equation may be degenerate or nondenerate Kirchhoff equation, and derive the blow up properties of solutions of this problem with negative and positive initial energy by the method different from the references [5]-[13].

The content of this paper is organized as follows. In Section 2, we give some lemmas. In Section 3, we prove the existence and uniqueness of the local solution by the Banach contraction mapping principle. In Section 4, we study the blow-up properties of solution for positive and negative initial energy and estimate for blow-up time $T^{*}$ by lemma of [9].

\section{Preliminaries}

In this section, we introduce material needed in the proof our main result. We use the standard Lebesgue space $L^{p}(\Omega)$ and Sobolev space $H^{m}(\Omega)$ with their usual scalar products and norms. Meanwhile we define $H_{0}^{m}(\Omega)=\left\{u \in H^{m}(\Omega): \frac{\partial^{i} u}{\partial v^{i}}=0, i=0,1, \cdots, m-1\right\}$ and introduce the following abbreviations: $\quad\|\cdot\|_{H^{m}}=\|\cdot\|_{H^{m}(\Omega)},\|\cdot\|_{H_{0}^{m}}=\|\cdot\|_{H_{0}^{m}(\Omega)},\|\cdot\|=\|\cdot\|_{L^{2}(\Omega)},\|\cdot\|_{p}=\|\cdot\|_{L^{p}(\Omega)} \quad$ for any real number $p>1$.

Lemma 2.1 (Sobolev-Poincaré inequality [8]) Let $s$ be a number with $2 \leq s<+\infty, n \leq 2 m$ and $2 \leq s \leq \frac{2 m}{n-2 m}, n>2 m$. Then there is a constant $K$ depending on $\Omega$ and $s$ such that

$$
\|u\|_{s} \leq K\left\|(-\Delta)^{\frac{m}{2}} u\right\|, \quad \forall u \in H_{0}^{m}(\Omega)
$$

Lemma 2.2 [9] Suppose that $\delta>0$ and $B(t)$ is a nonnegative $C^{2}(0,+\infty)$ function such that

$$
B^{\prime \prime}(t)-4(\delta+1) B^{\prime}(t)+4(\delta+1) B(t) \geq 0
$$

If

$$
B^{\prime}(0)>r_{2} B(0)+K_{0}
$$


then we have $\forall t>0, B^{\prime}(t)>K_{0}$. Here, $K_{0}$ is a constant and $r_{2}=2(\delta+1)-2 \sqrt{(\delta+1) \delta}$ the smallest positive root of the equation $r^{2}-4(\delta+1) r+4(\delta+1)=0$.

Lemma 2.3 [9] If $J(t)$ is a non-increasing function on $\left[t_{0},+\infty\right), t_{0} \geq 0$ such that

$$
J^{\prime}(t)^{2} \geq a+b J(t)^{2+\frac{1}{\delta}}, \forall t_{0} \geq 0,
$$

where $a>0, b \in R$. Then there exists a finite time $T^{*}$ such that $\lim _{t \rightarrow T^{*-}} J(t)=0$.

Moreover, for the case that $b<0, J\left(t_{0}\right)<\min \left\{1, \sqrt{\frac{a}{-b}}\right\}$, an upper bound of $T^{*}$ is $t_{0}+\frac{1}{\sqrt{-b}} \ln \frac{\sqrt{\frac{a}{-b}}}{\sqrt{\frac{a}{-b}}-J\left(t_{0}\right)}$;

If $b=0$, we have $T^{*} \leq t_{0}+\frac{J\left(t_{0}\right)}{\sqrt{a}}$;

If $b>0$, we have $T^{*} \leq \frac{J\left(t_{0}\right)}{\sqrt{a}}$ or $T^{*} \leq t_{0}+2^{\frac{3 \delta+1}{2 \delta}} \frac{\delta c}{\sqrt{a}}\left\{1-\left[1+c J\left(t_{0}\right)\right]^{\frac{-1}{2 \delta}}\right\}$.

\section{Local Existence of Solution}

Theorem 3.1 Suppose that $0 \leq p \leq \frac{4 m}{n-2 m} \quad(0 \leq p<+\infty$ if $0 \leq n \leq 2 m)$ and for any given $\left(u_{0}, u_{1}\right) \in H^{2 m}(\Omega) \cap H_{0}^{m}(\Omega) \times L^{2}(\Omega)$, then there exists $T>0$ such that the problem (1.1)-(1.3) has a unique local solution satisying

$$
\begin{aligned}
& u \in C^{0}\left([0, T] ; H^{2 m}(\Omega) \cap H_{0}^{m}(\Omega)\right), \\
& u_{t} \in C^{0}\left([0, T] ; L^{2}(\Omega)\right) \cap L^{2}\left(0, T ; H_{0}^{m}(\Omega)\right) .
\end{aligned}
$$

Proof. We proof the theorem by Banach contraction mapping principle. For $T>0$ and $R>0$, we define the following two-parameter space of solutions:

$$
X_{T, R} \equiv\left\{\begin{array}{l}
v \in C^{0}\left([0, T) ; H^{2 m}(\Omega) \cap H_{0}^{m}(\Omega)\right), \\
v_{t} \in C^{0}\left([0, T) ; L^{2}(\Omega)\right) \cap L^{2}\left(0, T ; H_{0}^{m}(\Omega)\right): \\
e_{1}(v(t)) \leq R^{2}, t \in[0, T], v(0)=u_{0}, v_{t}(0)=u_{1}
\end{array}\right\},
$$

where $e_{1}(v(t))=\left\|D^{2 m} v\right\|^{2}+\left\|v_{t}\right\|^{2}$. Then $X_{T, R}$ is a complete metric space with the distance

$$
d\left(v_{1}, v_{2}\right)=\sup _{0 \leq t \leq T} e_{1}\left(v_{1}(t)-v_{2}(t)\right) .
$$

We define the non-linear mapping $S$ in the following way. For $v \in X_{T, R}$, $u=S v$ is the unique solution of the following equation:

$$
u_{t t}+(-\Delta)^{m} u_{t}+\left(a+b\left\|D^{m} v\right\|^{2 q}\right)(-\Delta)^{m} u=|v|^{p} v
$$




$$
\begin{aligned}
& u(x, t)=0, \frac{\partial^{i} u}{\partial v^{i}}=0, i=1,2, \cdots, m-1, x \in \partial \Omega, t \in(0,+\infty), \\
& u(x, 0)=u_{0}(x), u_{t}(x, 0)=u_{1}(x), x \in \Omega .
\end{aligned}
$$

We shall show that there exist $T>0$ and $R>0$ such that

1) $S$ maps $X_{T, R}$ into itself;

2) $S$ is a contraction mapping with respect to the metric $d(\cdot, \cdot)$.

First, we shall check (i). Multiplying Equation (3.4) by $2 u_{t}+\frac{2}{3}(-\Delta)^{m} u$, and integrating it over $\Omega$, we have

$$
\begin{aligned}
& \frac{\mathrm{d} e_{2}(u(t))}{\mathrm{d} t}+\frac{4}{3}\left\|D^{m} u_{t}\right\|^{2}+\frac{2}{3}\left(a+b\left\|D^{m} v\right\|^{2 q}\right)\left\|D^{2 m} u\right\|^{2} \\
& =\left\|D^{m} u\right\|^{2} \frac{\mathrm{d}}{\mathrm{d} t}\left(a+b\left\|D^{m} v\right\|^{2 q}\right)+\left(|v|^{p} v, 2 u_{t}+\frac{2}{3}(-\Delta)^{m} u\right) \\
& \equiv I_{1}+I_{2},
\end{aligned}
$$

where $e_{2}(u(t))=\left\|u_{t}\right\|^{2}+\left(a+b\left\|D^{m} v\right\|^{2 q}\right)\left\|D^{m} u\right\|^{2}+\frac{1}{3}\left\|D^{2 m} u\right\|^{2}+\frac{2}{3}\left(u_{t}, D^{2 m} u\right)$.

To proceed the estimation,we observe that for $v \in X_{T, R}$. By Lemma 2.1, we have

$$
\begin{aligned}
I_{1} & =\left\|D^{m} u\right\|^{2} \frac{\mathrm{d}}{\mathrm{d} t}\left(a+b\left\|D^{m} v\right\|^{2 q}\right) \\
& =b\left\|D^{m} u\right\|^{2} \frac{\mathrm{d}}{\mathrm{d} t}\left\|D^{m} v\right\|^{2 q} \\
& =2 b q\left\|D^{m} u\right\|^{2}\left\|D^{m} v\right\|^{2 q-2}\left(D^{2 m} v, v_{t}\right) \\
& \leq 2 b q K^{2 q-2} R^{2 q-2}\left\|D^{2 m} v\right\|\left\|v_{t}\right\|\left\|D^{m} u\right\|^{2} \\
& \leq 2 b q K^{2 q} R^{2 q}\left\|D^{2 m} u\right\|^{2} \\
& \leq 2 b q K^{2 q} R^{2 q} e_{1}(u(t)) .
\end{aligned}
$$

Because of $0 \leq p \leq \frac{4 m}{n-2 m}(0 \leq p<+\infty$ if $0 \leq n \leq 2 m)$, then

$$
\begin{aligned}
I_{2} & =\left(|v|^{p} v, 2 u_{t}+\frac{2}{3}(-\Delta)^{m} u\right) \\
& \leq\left. 2\left|\int_{\Omega}\right| v\right|^{p} v u_{t} \mathrm{~d} x\left|+\frac{2}{3}\right| \int_{\Omega}|v|^{p} v \cdot D^{2 m} u \mathrm{~d} x \mid \\
& \leq 2\left\|u_{t}\right\|\|v\|_{2 p+2}^{p+1}+\frac{2}{3}\left\|D^{2 m} u\right\|\|v\|_{2 p+2}^{p+1} \\
& \leq 2 K^{2 p+2} R^{p+1}\left\|u_{t}\right\|+\frac{2}{3} K^{2 p+2} R^{p+1}\left\|D^{2 m} u\right\| \\
& \leq 2 K^{2 p+2} R^{p+1}\left(e_{1}(u(t))\right)^{\frac{1}{2}}+\frac{2}{3} K^{2 p+2} R^{p+1}\left(e_{1}(u(t))\right)^{\frac{1}{2}} \\
& \leq 4 K^{2 p+2} R^{p+1}\left(e_{1}(u(t))\right)^{\frac{1}{2}} .
\end{aligned}
$$

Since $\frac{2}{3}\left(u_{t}, D^{2 m} u\right) \geq-\frac{2}{3}\left\|u_{t}\right\|^{2}-\frac{1}{6}\left\|D^{2 m} u\right\|^{2}$ by the Young inequality, we see that 


$$
e_{2}(u(t)) \geq \frac{1}{3}\left\|u_{t}\right\|^{2}+\frac{1}{6}\left\|D^{2 m} u\right\|^{2} \geq \frac{1}{6} e_{1}(u(t)) .
$$

Combining these inequalities, we get

$$
\frac{\mathrm{d} e_{2}(u(t))}{\mathrm{d} t}+\frac{4}{3}\left\|D^{m} u_{t}\right\|^{2} \leq 12 b q K^{2 q} R^{2 q} e_{2}(u(t))+12 K^{2 p+2} R^{p+1}\left(e_{2}(u(t))\right)^{\frac{1}{2}} .
$$

Therefore, by the Gronwall inequality, we obtain

$$
e_{2}(u(t))+\frac{4}{3} \int_{0}^{T}\left\|D^{m} u_{t}(s)\right\|^{2} \mathrm{~d} s \leq\left[\left(e_{2}(u(0))\right)^{\frac{1}{2}}+12 K^{2 p+2} R^{p+1} T\right]^{2} e^{12 b q K^{2 q} R^{2 q} T},
$$

where $e_{2}(u(0))=\left\|u_{1}\right\|^{2}+\left(a+b\left\|D^{m} u_{0}\right\|^{2 q}\right)\left\|D^{m} u_{0}\right\|^{2}+\frac{1}{3}\left\|D^{2 m} u_{0}\right\|^{2}+\frac{2}{3}\left(u_{1}, D^{2 m} u_{0}\right)$ and

$$
e_{2}(u(0)) \leq 2\left\|u_{1}\right\|^{2}+\left(1+a+b\left\|D^{m} u_{0}\right\|^{2 q}\right)\left\|D^{m} u_{0}\right\|^{2} .
$$

So, for all $t \in[0, T]$, we obtain

$$
\begin{aligned}
& e_{1}(u(t))+\frac{4}{3} \int_{0}^{T}\left\|D^{m} u_{t}(s)\right\|^{2} \mathrm{~d} s \\
& \leq 6 e_{2}(u(t))+8 \int_{0}^{T}\left\|D^{m} u_{t}(s)\right\|^{2} \mathrm{~d} s \\
& \leq 6\left[\left(2\left\|u_{1}\right\|^{2}+\left(1+a+b\left\|D^{m} u_{0}\right\|^{2 q}\right)\left\|D^{m} u_{0}\right\|^{2}\right)^{\frac{1}{2}}+12 K^{2 p+2} R^{p+1} T\right]^{2} e^{12 b q K^{2 q} R^{2 q} T} .
\end{aligned}
$$

Therefore, in order that the map $S$ verifies 1), it will be enough that the parameters $T$ and $R$ satisfy

$$
\begin{aligned}
& 6\left[\left(2\left\|u_{1}\right\|^{2}+\left(1+a+b\left\|D^{m} u_{0}\right\|^{2 q}\right)\left\|D^{m} u_{0}\right\|^{2}\right)^{\frac{1}{2}}+12 K^{2 p+2} R^{p+1} T\right]^{2} e^{12 b q K^{2 q} R^{2 q} T} \\
& \leq R^{2} .
\end{aligned}
$$

Moreover, it follows from (3.14) that $u_{t} \in L^{\infty}\left(0, T ; L^{2}(\Omega)\right) \cap L^{2}\left(0, T ; H_{0}^{m}(\Omega)\right)$ and $u \in L^{\infty}\left(0, T ; H^{2 m}(\Omega) \cap H_{0}^{m}(\Omega)\right)$. It implies

$$
\begin{aligned}
& u \in C^{0}\left([0, T] ; H^{2 m}(\Omega) \cap H_{0}^{m}(\Omega)\right), \\
& u_{t} \in C^{0}\left([0, T] ; L^{2}(\Omega)\right) \cap L^{2}\left(0, T ; H_{0}^{m}(\Omega)\right) .
\end{aligned}
$$

Next, we prove 2). Suppose that (3.15) holds. We take $v_{1}, v_{2} \in X_{T, R}$, let $u_{1}=S v_{1}, u_{2}=S v_{2}$, and set $w=u_{1}-u_{2}$. Then $w$ satisfies

$$
\begin{aligned}
& w_{t t}+(-\Delta)^{m} w_{t}+\left(a+b\left\|D^{m} v_{1}\right\|^{2 q}\right)(-\Delta)^{m} w \\
& =-b\left(\left\|D^{m} v_{1}\right\|^{2 q}-\left\|D^{m} v_{2}\right\|^{2 q}\right)(-\Delta)^{m} u_{2}+\left(\left|v_{1}\right|^{p} v_{1}-\left|v_{2}\right|^{p} v_{2}\right), \\
& (x, t) \in \Omega \times[0, T], \\
& w(x, t)=\frac{\partial^{i} w}{\partial v^{i}}=0, \quad(x, t) \in \partial \Omega \times[0, T], \\
& w(x, 0)=0, \quad w_{t}(x, 0)=0, \quad x \in \Omega .
\end{aligned}
$$


Multiplying (3.17-3.18) by $2 w_{t}$ and integrating it over $\Omega$ and using Green's formula, we have

$$
\begin{aligned}
& \frac{\mathrm{d}}{\mathrm{d} t}\left[\left\|w_{t}\right\|^{2}+\left(a+b\left\|D^{m} v_{1}\right\|^{2 q}\right)\left\|D^{m} w\right\|^{2}\right]+2\left\|D^{m} w_{t}\right\|^{2} \\
& =\left\|D^{m} w\right\|^{2} \frac{\mathrm{d}}{\mathrm{d} t}\left(a+b\left\|D^{m} v_{1}\right\|^{2 q}\right)-2 b\left(\left\|D^{m} v_{1}\right\|^{2 q}-\left\|D^{m} v_{2}\right\|^{2 q}\right)\left(D^{2 m} u_{2}, w_{t}\right) \\
& \quad+2\left(\left|v_{1}\right|^{p} v_{1}-\left|v_{2}\right|^{p} v_{2}, w_{t}\right) \\
& \equiv I_{3}+I_{4}+I_{5} .
\end{aligned}
$$

To proceed the estimation, by Lemma 2.1 observe that

$$
\begin{aligned}
I_{3}= & \left\|D^{m} w\right\|^{2} \frac{\mathrm{d}}{\mathrm{d} t}\left(a+b\left\|D^{m} v_{1}\right\|^{2 q}\right) \leq 2 b q K^{2 q} R^{2 q} e_{1}(w(t)) . \\
I_{4}= & -2 b\left(\left\|D^{m} v_{1}\right\|^{2 q}-\left\|D^{m} v_{2}\right\|^{2 q}\right)\left(D^{2 m} u_{2}, w_{t}\right) \\
\leq & 2 b q\left[\theta\left\|D^{m} v_{1}\right\|^{2}+(1-\theta)\left\|D^{m} v_{2}\right\|^{2}\right]^{q-1} \\
& \times\left(\left\|D^{m} v_{1}\right\|+\left\|D^{m} v_{2}\right\|\right)\left\|D^{m}\left(v_{1}-v_{2}\right)\right\|\left\|D^{2 m} u_{2}\right\|\left\|w_{t}\right\| \\
\leq & 4 b q K^{2 q} R^{2 q}\left[e_{1}\left(v_{1}(t)-v_{2}(t)\right)\right]^{\frac{1}{2}}\left[e_{1}(w(t))\right]^{\frac{1}{2}}
\end{aligned}
$$

where $0<\theta<1$.

$$
\begin{aligned}
I_{5} & =2\left(\left|v_{1}\right|^{p} v_{1}-\left|v_{2}\right|^{p} v_{2}, w_{t}\right) \\
& \leq 2 \int_{\Omega}\left(\left|v_{1}\right|^{p}+\left|v_{2}\right|^{p}\right)\left|v_{1}-v_{2} \| w_{t}\right| \mathrm{d} x \\
& \leq 2\left(\left\|v_{1}\right\|_{n p}^{p}+\left\|v_{2}\right\|_{n p}^{p}\right)\left\|v_{1}-v_{2}\right\|_{\frac{2 n}{n-2}}\left\|w_{t}\right\| \\
& \leq 4 K^{2 p+2} R^{p}\left\|D^{2 m}\left(v_{1}-v_{2}\right)\right\|\left\|w_{t}\right\| \\
& \leq 4 K^{2 p+2} R^{p}\left[e_{1}\left(v_{1}(t)-v_{2}(t)\right)\right]^{\frac{1}{2}}\left[e_{1}(w(t))\right]^{\frac{1}{2}} .
\end{aligned}
$$

Substituting (3.22)-(3.24) into (3.21), we obtain

$$
\begin{aligned}
& \frac{\mathrm{d}}{\mathrm{d} t}\left[\left\|w_{t}\right\|^{2}+\left(a+b\left\|D^{m} v_{1}\right\|^{2 q}\right)\left\|D^{m} w\right\|^{2}\right]+2\left\|D^{m} w_{t}\right\|^{2} \\
& \leq 2 b q K^{2 q} R^{2 q} e_{1}(w(t)) \\
& \quad+\left(4 b q K^{2 q} R^{2 q}+4 K^{2 p+2} R^{p}\right)\left[e_{1}\left(v_{1}(t)-v_{2}(t)\right)\right]^{\frac{1}{2}}\left[e_{1}(w(t))\right]^{\frac{1}{2}} .
\end{aligned}
$$

According to the same method, Multiplying (3.17-3.18) by $2 \mathrm{Aw}$ and integrating it over $\Omega$, we get

$$
\begin{aligned}
& \frac{\mathrm{d}}{\mathrm{d} t}\left[\left\|D^{2 m} w\right\|^{2}+2\left(w_{t}, D^{2 m} w\right)\right]+2\left(a+b\left\|D^{m} v_{1}\right\|^{2 q}\right)\left\|D^{2 m} w\right\|^{2} \\
& =2\left\|D^{m} w_{t}\right\|^{2}-2 b\left(\left\|D^{m} v_{1}\right\|^{2 q}-\left\|D^{m} v_{2}\right\|^{2 q}\right)\left(D^{2 m} u_{2}, D^{2 m} w\right) \\
& \quad+2\left(\left|v_{1}\right|^{p} v_{1}-\left|v_{2}\right|^{p} v_{2}, D^{2 m} w\right) \\
& \leq 2\left\|D^{m} w_{t}\right\|^{2}+\left(4 b q K^{2 q} R^{2 q}+4 K^{2 p+2} R^{p}\right)\left[e_{1}\left(v_{1}(t)-v_{2}(t)\right)\right]^{\frac{1}{2}}\left[e_{1}(w(t))\right] .
\end{aligned}
$$


Taking (3.25) $+\frac{1}{3} \times(3.26)$ and by (3.10), it follows that

$$
\begin{aligned}
& \frac{\mathrm{d} e_{2}(w(t))}{\mathrm{d} t} \\
& \leq 2 b q K^{2 q} R^{2 q} e_{1}(w(t)) \\
& \quad+\left(8 b q K^{2 q} R^{2 q}+8 K^{2 p+2} R^{p}\right)\left[e_{1}\left(v_{1}(t)-v_{2}(t)\right)\right]^{\frac{1}{2}}\left[e_{1}(w(t))\right]^{\frac{1}{2}} \\
& \leq 2 b q K^{2 q} R^{2 q} e_{2}(w(t)) \\
& \quad+\left(72 b q K^{2 q} R^{2 q}+72 K^{2 p+2} R^{p}\right)\left[e_{2}\left(v_{1}(t)-v_{2}(t)\right)\right]^{\frac{1}{2}}\left[e_{2}(w(t))\right]^{\frac{1}{2}},
\end{aligned}
$$

where $e_{2}(w(t))=\left\|w_{t}\right\|^{2}+\left(a+b\left\|D^{m} v_{1}\right\|^{2 q}\right)\left\|D^{m} w\right\|^{2}+\frac{1}{3}\left\|D^{2 m} w\right\|^{2}+\frac{2}{3}\left(w_{t}, D^{2 m} w\right)$ and $e_{2}(w(0))=0$.

Applying the Gronwall inequality, we have

$$
e_{2}(w(t)) \leq\left(72 b q K^{2 q} R^{2 q}+72 K^{2 p+2} R^{p}\right)^{2} T^{2} e^{2 b q K^{2 q} R^{2 q} T} \sup _{0 \leq t \leq T} e_{1}\left(v_{1}(t)-v_{2}(t)\right) .
$$

So, by (3.10) we have

$$
\sup _{0 \leq t \leq T} e_{1}\left(u_{1}(t)-u_{2}(t)\right) \leq C_{T, R} \sup _{0 \leq t \leq T} e_{1}\left(v_{1}(t)-v_{2}(t)\right),
$$

where $C_{T, R} \equiv\left(72 \sqrt{6} b q K^{2 q} R^{2 q}+72 \sqrt{6} K^{2 p+2} R^{p}\right)^{2} T^{2} e^{2 b q K^{2 q} R^{2 q} T}$. If $C_{T, R}<1$, we can see $S$ is a contraction mapping. Finally, we choose suitable $R$ is sufficiently large and $T$ is sufficiently small, such that 1) and 2) hold. By applying Banach fixed point theorem, we obtain the local existence.

\section{Blow-Up of Solution}

In this section, we shall discuss the blow-up properties for the problem (1.1)(1.3). For this purpose, we give the following definition and lemmas.

Now, we define the energy function of the solution $u$ of (1.1)-(1.3) by

$$
E(t)=\frac{1}{2}\left\|u_{t}\right\|^{2}+\frac{a}{2}\left\|D^{m} u\right\|^{2}+\frac{b}{2 q+2}\left\|D^{m} u\right\|^{2 q+2}-\frac{1}{p+2}\|u\|_{p+2}^{p+2}, t \geq 0 .
$$

Then, we have

$$
E(t)=E(0)-\int_{0}^{t}\left\|D^{m} u_{t}(s)\right\|^{2} \mathrm{~d} s,
$$

where $E(0)=\frac{1}{2}\left\|u_{1}\right\|^{2}+\frac{a}{2}\left\|D^{m} u_{0}\right\|^{2}+\frac{b}{2 q+2}\left\|D^{m} u_{0}\right\|^{2 q+2}-\frac{1}{p+2}\left\|u_{0}\right\|_{p+2}^{p+2}$.

Definition 4.1 A solution $u(t)$ of (1.1)-(1.3) is called a blow-up solution, if there exists a finite time $T^{*}$ such that

$$
\lim _{t \rightarrow T^{*-}} \int_{\Omega}\left|\nabla^{m} u\right|^{2} \mathrm{~d} x=+\infty
$$

For the next lemma, we define

$$
F(t):=F(u(t))=\|u(t)\|^{2}+\int_{0}^{t}\left\|D^{m} u(s)\right\|^{2} \mathrm{~d} s .
$$

Lemma 4.1 Suppose that $0 \leq p \leq \frac{4 m}{n-2 m}(0 \leq p<+\infty$ if $0 \leq n \leq 2 m)$ and 
$p \geq 2 q$ hold. Then we have the following results, which are

1) $F^{\prime \prime}(t)-(p+4)\left\|u_{t}\right\|^{2} \geq-(4+2 p) E(0)+(4+2 p) \int_{0}^{t}\left\|D^{m} u_{t}(s)\right\|^{2} \mathrm{~d} s$, for $t \geq$ 0 ;

2) If $E(0)<0$, we get $F^{\prime}(t)>\left\|D^{m} u_{0}\right\|^{2}$ for $t>t^{*}$, where $t^{*}=\max \left\{0, \frac{F^{\prime}(0)-\left\|D^{m} u_{0}\right\|^{2}}{(4+2 p) E(0)}\right\}$;

3) If $E(0)=0$ and if $F^{\prime}(0)>\left\|D^{m} u_{0}\right\|^{2}$, i.e. $\int_{\Omega} u_{0} u_{1} \mathrm{~d} x>0$ hold, then we have $F^{\prime}(t)>\left\|D^{m} u_{0}\right\|^{2}$ for $t \geq 0$;

4) If $E(0)>0$ and

$F^{\prime}(0)>\left(\frac{4+p-\sqrt{p^{2}+4 p}}{2}\right)\left[\left(F(0)+\frac{(4+2 p) E(0)+(4+p)\left\|D^{m} u_{0}\right\|^{2}}{4+p}\right)\right]+\left\|D^{m} u_{0}\right\|^{2}$ hold, then we get $F^{\prime}(t)>\left\|D^{m} u_{0}\right\|^{2}$ for $t>0$.

Proof. Step 1: From (4.4), we obtain

$$
F^{\prime}(t)=2\left(u, u_{t}\right)+\left\|D^{m} u(t)\right\|^{2},
$$

and

$$
\begin{aligned}
& F^{\prime \prime}(t) \\
& =2\left\|u_{t}\right\|^{2}+2\left(u, u_{t t}\right)+\frac{\mathrm{d}}{\mathrm{d} t}\left\|D^{m} u\right\|^{2} \\
& =2\left\|u_{t}\right\|^{2}+2\left(u,-(-\Delta)^{m} u_{t}-\left(a+b\left\|D^{m} u\right\|^{2 q}\right)(-\Delta)^{m} u+|u|^{p} u\right)+\frac{\mathrm{d}}{\mathrm{d} t}\left\|D^{m} u\right\|^{2} \\
& =2\left\|u_{t}\right\|^{2}-2\left(a+b\left\|D^{m} u\right\|^{2 q}\right)\left\|D^{m} u\right\|^{2}+2\|u\|_{p+2}^{p+2} .
\end{aligned}
$$

From the above equation and the energy identity and $p \geq 2 q$, we obtain

$$
\begin{aligned}
& F^{\prime \prime}(t)-(4+p)\left\|u_{t}\right\|^{2} \\
&=-(p+2)\left\|u_{t}\right\|^{2}-2\left(a+b\left\|D^{m} u\right\|^{2 q}\right)\left\|D^{m} u\right\|^{2}+2\|u\|_{p+2}^{p+2} \\
&=-(p+2)\left(2 E(0)-2 \int_{0}^{t}\left\|D^{m} u_{t}(s)\right\|^{2} \mathrm{~d} s-a\left\|D^{m} u\right\|^{2}-\frac{b}{q+1}\left\|D^{m} u\right\|^{2 q+2}+\frac{2}{p+2}\|u\|_{p+2}^{p+2}\right) \\
&-2\left(a+b\left\|D^{m} u\right\|^{2 q}\right)\left\|D^{m} u\right\|^{2}+2\|u\|_{p+2}^{p+2} \\
&=-(2 p+4) E(0)+(2 p+4) \int_{0}^{t}\left\|D^{m} u_{t}(s)\right\|^{2} \mathrm{~d} s+a p\left\|D^{m} u\right\|^{2}+\frac{b(p-2 q)}{q+1}\left\|D^{m} u\right\|^{2 q+2} \\
& \geq-(2 p+4) E(0)+(2 p+4) \int_{0}^{t}\left\|D^{m} u_{t}(s)\right\|^{2} \mathrm{~d} s .
\end{aligned}
$$

Therefore, we obtain 1$)$.

Step 2: If $E(0)<0$, then by (i), we have

$$
F^{\prime \prime}(t) \geq-(2 p+4) E(0) .
$$

Integrating (4.8) over $[0, t]$, we have that

$$
F^{\prime}(t) \geq F^{\prime}(0)-(2 p+4) E(0) t, t \geq 0 .
$$


Thus, we get $F^{\prime}(t)>\left\|D^{m} u_{0}\right\|^{2}$ for $t>t^{*}$, where $t^{*}=\max \left\{0, \frac{F^{\prime}(0)-\left\|D^{m} u_{0}\right\|^{2}}{(4+2 p) E(0)}\right\}$.

So, 2) has been proved.

Step 3: If $E(0)=0$, then for $t \geq 0$ we have

$$
F^{\prime \prime}(t) \geq(p+4)\left\|u_{t}\right\|^{2}+(2 p+4) \int_{0}^{t}\left\|D^{m} u_{t}(s)\right\|^{2} \mathrm{~d} s \geq 0 .
$$

Integrating (4.10) over $[0, t]$, we have that

$$
F^{\prime}(t) \geq F^{\prime}(0) \text {. }
$$

And because of $F^{\prime}(0)>\left\|D^{m} u_{0}\right\|^{2}$, i.e. $\int_{\Omega} u_{0} u_{1} \mathrm{~d} x>0$, then we get $F^{\prime}(t) \geq F^{\prime}(0)=2\left(u_{0}, u_{1}\right)+\left\|D^{m} u_{0}\right\|^{2}>\left\|D^{m} u_{0}\right\|^{2}$.

Thus, 3) has been proved.

Step 4: For the case that $E(0)>0$, we first note that

$$
2 \int_{0}^{t}\left(D^{m} u, D^{m} u_{t}\right) \mathrm{d} t=\int_{0}^{t} \frac{\mathrm{d}}{\mathrm{d} t}\left\|D^{m} u\right\|^{2} \mathrm{~d} t=\left\|D^{m} u\right\|^{2}-\left\|D^{m} u_{0}\right\|^{2} .
$$

By using Hölder inequality, we have

$$
\begin{aligned}
\left\|D^{m} u\right\|^{2} & =\left\|D^{m} u_{0}\right\|^{2}+\int_{0}^{t}\left(D^{m} u, D^{m} u_{t}\right) \mathrm{d} t \\
& \leq\left\|D^{m} u_{0}\right\|^{2}+\int_{0}^{t}\left\|D^{m} u\right\|^{2} \mathrm{~d} t+\int_{0}^{t}\left\|D^{m} u_{t}\right\|^{2} \mathrm{~d} t .
\end{aligned}
$$

So

$$
\begin{aligned}
F^{\prime}(t) & =2\left(u, u_{t}\right)+\left\|D^{m} u\right\|^{2} \\
& \leq\|u\|^{2}+\left\|u_{t}\right\|^{2}+\left\|D^{m} u\right\|^{2} \\
& \leq\|u\|^{2}+\left\|u_{t}\right\|^{2}+\left\|D^{m} u_{0}\right\|^{2}+\int_{0}^{t}\left\|D^{m} u\right\|^{2} \mathrm{~d} t+\int_{0}^{t}\left\|D^{m} u_{t}\right\|^{2} \mathrm{~d} t \\
& =F(t)+\left\|u_{t}\right\|^{2}+\left\|D^{m} u_{0}\right\|^{2}+\int_{0}^{t}\left\|D^{m} u_{t}\right\|^{2} \mathrm{~d} t .
\end{aligned}
$$

Thus, we have

$$
F^{\prime \prime}(t)-(4+p) F^{\prime}(t)+(4+p) F(t)+K_{1} \geq p \int_{0}^{t}\left\|D^{m} u_{t}\right\|^{2} \mathrm{~d} t \geq 0,
$$

where $K_{1}=(4+2 p) E(0)+(4+p)\left\|D^{m} u_{0}\right\|^{2}$.

Set

$$
B(t)=F(t)+\frac{K_{1}}{4+p}, t>0 .
$$

Then $B(t)$ satisfies (2.2). By conditions

$F^{\prime}(0)>\left(\frac{4+p-\sqrt{p^{2}+4 p}}{2}\right)\left[\left(F(0)+\frac{(4+2 p) E(0)+(4+p)\left\|D^{m} u_{0}\right\|^{2}}{4+p}\right]+\left\|D^{m} u_{0}\right\|^{2}\right.$ and Lemma 2.2, then $F^{\prime}(t)>\left\|D^{m} u_{0}\right\|^{2}$ for $t>0$.

Lemma 4.2 Suppose that $0 \leq p \leq \frac{4 m}{n-2 m} \quad(0 \leq p<+\infty$ if $0 \leq n \leq 2 m)$ and $p \geq 2 q$ hold and that eigher one of the following conditions is satisfied: 
1) $E(0)<0$;

2) $E(0)=0$ and $\int_{\Omega} u_{0} u_{1} \mathrm{~d} x>0$;

3) $E(0)>0$ and

$F^{\prime}(0)>\left(2+\frac{p}{2}-\frac{\sqrt{p^{2}+4 p}}{2}\right)\left[\left(F(0)+\frac{(4+2 p) E(0)+(4+p)\left\|D^{m} u_{0}\right\|^{2}}{4+p}\right)\right]+\left\|D^{m} u_{0}\right\|^{2}$

hold.

Then, there exists $t_{0} \geq 0$, such that $F^{\prime}(t)>\left\|D^{m} u_{0}\right\|^{2}$ for $t>t_{0}$.

Proof. By Lemma 4.1, $t_{0}=t^{*}$ in case (i) and $t_{0}=0$ in case 2) and 3).

Theorem 4.1 Suppose that $0 \leq p \leq \frac{4 m}{n-2 m} \quad(0 \leq p<+\infty$ if $0 \leq n \leq 2 m)$ and $p \geq 2 q$ hold and that eigher one of the following conditions is satisfied:

1) $E(0)<0$;

2) $E(0)=0$ and $\int_{\Omega} u_{0} u_{1} \mathrm{~d} x>0$;

3) $0<E(0)<\frac{\left(F^{\prime}\left(t_{0}\right)-\left\|D^{m} u_{0}\right\|^{2}\right)^{2}}{8\left[F\left(t_{0}\right)+\left(T_{1}-t_{0}\right)\left\|D^{m} u_{0}\right\|^{2}\right]}$ and

$F^{\prime}(0)>\left(\frac{4+p-\sqrt{p^{2}+4 p}}{2}\right)\left[\left(F(0)+\frac{(4+2 p) E(0)+(4+p)\left\|D^{m} u_{0}\right\|^{2}}{4+p}\right)\right]+\left\|D^{m} u_{0}\right\|^{2}$

hold.

Then the solution $u$ blow up at finite $T^{*}$. And $T^{*}$ can be estimated by (4.26)-(4.29), respectively, according to the sign of $E(0)$.

Proof. Let

$$
J(t)=\left(F(t)+\left(T_{1}-t\right)\left\|D^{m} u_{0}\right\|^{2}\right)^{-\frac{p}{4}}, t \in\left[0, T_{1}\right],
$$

where $T_{1}$ is some certain constant which will be chosen later. Then we get

$$
J^{\prime}(t)=-\frac{p}{4} J(t)^{1+\frac{4}{p}}\left(F^{\prime}(t)-\left\|D^{m} u_{0}\right\|^{2}\right),
$$

and

$$
J^{\prime \prime}(t)=-\frac{p}{4} J(t)^{1+\frac{8}{p}} V(t),
$$

where $V(t)=F^{\prime \prime}(t)\left(F(t)+\left(T_{1}-t\right)\left\|D^{m} u_{0}\right\|^{2}\right)-\frac{4+p}{4}\left(F^{\prime}(t)-\left\|D^{m} u_{0}\right\|^{2}\right)^{2}$.

By the Hölder inequality, we obtain

$$
\begin{aligned}
F^{\prime}(t) & =2\left(u, u_{t}\right)+\left\|D^{m} u\right\|^{2} \\
& =2\left(u, u_{t}\right)+\left\|D^{m} u_{0}\right\|^{2}+2 \int_{0}^{t}\left(D^{m} u, D^{m} u_{t}\right) \mathrm{d} t \\
& \leq 2\|u\| \cdot\left\|u_{t}\right\|+\left\|D^{m} u_{0}\right\|^{2}+2 \int_{0}^{t}\left\|D^{m} u\right\|^{2} \mathrm{~d} t \cdot \int_{0}^{t}\left\|D^{m} u_{t}\right\|^{2} \mathrm{~d} t \\
& =\left\|D^{m} u_{0}\right\|^{2}+2(\sqrt{P R}+\sqrt{Q S}),
\end{aligned}
$$

where $P=\|u(t)\|^{2}, Q=\int_{0}^{t}\|u(s)\|^{2} \mathrm{~d} s, R=\left\|u_{t}(t)\right\|^{2}, S=\int_{0}^{t}\left\|u_{t}(s)\right\|^{2} \mathrm{~d} s$. 
By 1) of Lemma 4.1, we get

$$
F^{\prime \prime}(t) \geq-(4+2 p) E(0)+(4+p)(R+S) .
$$

Then, we obtain

$$
\begin{aligned}
V(t) \geq & {[-(4+2 p) E(0)+(4+p)(R+S)]\left(F(t)+\left(T_{1}-t\right)\left\|D^{m} u_{0}\right\|^{2}\right) } \\
& -(4+p)(\sqrt{P R}+\sqrt{Q S})^{2} \\
= & -(4+2 p) E(0) J(t)^{-\frac{4}{p}}+(4+p)(R+S)\left(T_{1}-t\right)\left\|D^{m} u_{0}\right\|^{2} \\
& +(4+p)\left[(R+S)(P+Q)-(\sqrt{P R}+\sqrt{Q S})^{2}\right] \\
\geq & -(4+2 p) E(0) J(t)^{-\frac{4}{p}}, t \geq t_{0} .
\end{aligned}
$$

Therefore, we get

$$
J^{\prime \prime}(t) \leq\left(p+\frac{p^{2}}{2}\right) E(0) J(t)^{\frac{p+4}{p}}, t \geq t_{0} .
$$

Note that by Lemma 4.2, $J^{\prime}(t)<0, t>t_{0}$. Multiplying (4.23) by $J^{\prime}(t)$ and integrating it from $t_{0}$ to $t$, we have

$$
J^{\prime}(t)^{2} \geq \alpha+\beta J(t)^{\frac{2 p+4}{p}}, t \geq t_{0},
$$

where $\alpha=\frac{p^{2}}{16} J\left(t_{0}\right)^{\frac{2 p+4}{p}}\left[\left(F^{\prime}\left(t_{0}\right)-\left\|D^{m} u_{0}\right\|^{2}\right)^{2}-8 E(0) J\left(t_{0}\right)^{-\frac{4}{p}}\right]$, and $\beta=\frac{p^{2}}{2} E(0)$.

When $E(0)<0$ and $E(0)=0$, we obviously have $\alpha>0$. When $E(0)>0$, we also have $\alpha>0$ by condition $E(0)<\frac{\left(F^{\prime}\left(t_{0}\right)-\left\|D^{m} u_{0}\right\|^{2}\right)^{2}}{8\left[F\left(t_{0}\right)+\left(T_{1}-t_{0}\right)\left\|D^{m} u_{0}\right\|^{2}\right]}$.

Then by Lemma 2.3, there exists a finite time $T^{*}$ such that $\lim _{t \rightarrow T^{*-}} J(t)=0$ and the upper bounds of $T^{*}$ are estimated respectively according to the sign of $E(0)$. This will imply that

$$
\lim _{t \rightarrow T^{*-}}\left(\|u(t)\|^{2}+\int_{0}^{t}\left\|D^{m} u(s)\right\|^{2} \mathrm{~d} s\right)=+\infty
$$

Next, $T^{*}$ are estimated respectively according to the sign of $E(0)$ and

\section{Lemma 2.3.}

In case 1 ), we have

$$
T^{*} \leq t_{0}-\frac{J\left(t_{0}\right)}{J^{\prime}\left(t_{0}\right)}
$$

Furthermore, if $J\left(t_{0}\right)<\min \left\{1, \sqrt{\frac{\alpha}{-\beta}}\right\}$, then we have 


$$
T^{*} \leq t_{0}+\sqrt{\frac{1}{-\beta}} \ln \frac{\sqrt{\frac{\alpha}{-\beta}}}{\sqrt{\frac{\alpha}{-\beta}}-J\left(t_{0}\right)} .
$$

In case 2), we get

$$
T^{*} \leq t_{0}-\frac{J\left(t_{0}\right)}{J^{\prime}\left(t_{0}\right)} \text { or } T^{*} \leq t_{0}-\frac{J\left(t_{0}\right)}{\sqrt{\alpha}} .
$$

In case 3), we obtain

$$
T^{*} \leq \frac{J\left(t_{0}\right)}{\sqrt{\alpha}} \text { or } T^{*} \leq t_{0}+2^{\frac{3 p+4}{2 p}} \frac{p c}{4 \sqrt{\alpha}}\left\{1-\left[1+c J\left(t_{0}\right)\right]^{\frac{2}{p}}\right\},
$$

where $c=\left(\frac{\beta}{\alpha}\right)^{\frac{p}{p+8}}$. Note that in case 1), $t_{0}=t^{*}$ is given Lemma 4.1, and in case 2) and case 3) $t_{0}=0$.

Remark 4.1 [10] The choice of $T_{1}$ in (4.17) is possible under some conditions.

1) In the case $E(0)=0$, we can choose $T_{1} \geq \frac{16\left\|u_{0}\right\|^{2}}{p^{2} K^{2}\left\|u_{1}\right\|^{2}}$. In particular, we choose $T_{1}=\frac{16\left\|u_{0}\right\|^{2}}{p^{2} K^{2}\left\|u_{1}\right\|^{2}}$, then we get $T^{*} \leq \frac{16\left\|u_{0}\right\|^{2}}{p^{2} K^{2}\left\|u_{1}\right\|^{2}}$.

2) In the case $E(0)<0$, we can choose $T_{1}$ as in 1) if $\int_{\Omega} u_{0} u_{1} \mathrm{~d} x>0$ or $T_{1} \geq t^{*}-\frac{J\left(t_{0}\right)}{J^{\prime}\left(t_{0}\right)}$ if $\int_{\Omega} u_{0} u_{1} \mathrm{~d} x \leq 0$.

3) For the case $E(0)>0$. Under the condition $E(0)<\min \left\{k_{1}, k_{2}\right\}$, here $k_{1}=\frac{(4+p)\left[F^{\prime}(0)-\frac{4+p-\sqrt{p^{2}+4 p}}{2} F(0)-\frac{6+p-\sqrt{p^{2}+4 p}}{2}\left\|D^{m} u_{0}\right\|^{2}\right]}{\left(4+p-\sqrt{p^{2}+4 p}\right)(2+p)}$, $k_{2}=\frac{\left[4\left(\int_{\Omega} u_{0} u_{1} \mathrm{~d} x\right)^{2}-1\right]\left[p-4\left\|D^{m} u_{0}\right\|^{2}\right]}{8 p\left\|D^{m} u_{0}\right\|^{2}}$, if $\left\|D^{m} u_{0}\right\|^{2}<\frac{p}{4}, T_{1}$ is chosen to satisfy $k_{3} \leq T_{1} \leq k_{4}$, where $k_{3}=\frac{4\left\|u_{0}\right\|^{2}}{p-4\left\|D^{m} u_{0}\right\|^{2}}$, $k_{4}=\frac{4\left(\int_{\Omega} u_{0} u_{1} \mathrm{~d} x\right)^{2}-8 E(0)\left\|u_{0}\right\|^{2}-1}{8 E(0)\left\|D^{m} u_{0}\right\|^{2}}$. Therefore, we have $T \leq T^{*} \leq \frac{k_{3}}{\sqrt{4\left(\int_{\Omega} u_{0} u_{1} \mathrm{~d} x\right)^{2}-8 E(0) k_{3}}}$.

\section{Conclusion}

In this paper, we prove that nonlinear wave equations of higher-order Kirchhoff Type with Strong Dissipation exist unique local solution on 
$u \in C^{0}\left([0, T] ; H^{2 m}(\Omega) \cap H_{0}^{m}(\Omega)\right), u_{t} \in C^{0}\left([0, T] ; L^{2}(\Omega)\right) \cap L^{2}\left(0, T ; H_{0}^{m}(\Omega)\right)$. Then, we establish three blow-up results for certain solutions in the case 1$)$ : $E(0)<0$, in the case 2): $E(0)=0$ and in the case 3): $E(0)>0$. At last, we consider that the estimation of the upper bounds of the blow-up time $T^{*-}$ is given for deferent initial energy.

\section{Acknowledgements}

The authors express their sincere thanks to the anonymous reviewer for his/her careful reading of the paper, giving valuable comments and suggestions. These contributions greatly improved the paper.

This work is supported by the National Natural Sciences Foundation of People's Republic of China under Grant 11561076.

\section{References}

[1] Kirchhoff, G. (1883) Vorlesungen über Mechanik. Teubner, Leipzig.

[2] Ball, J.M. (1997) Remarks on Blow-Up and Nonexistence Theorems for Nonlinear Evolution Equations. The Quarterly Journal of Mathematics, Oxford Series, 28, 473486.

[3] Kopáčková, M. (1989) Remarks on Bounded Solutions of a Semilinear Dissipative Hyperbolic Equation. Commentationes Mathematicae Universitatis Carolinae, 30, 713-719.

[4] Haraux, A. and Zuazua, E. (1988) Decay Estimates for Some Semilinear Damped Hyperbolic Problems. Archive for Rational Mechanics and Analysis, 100, 191-206. https://doi.org/10.1007/BF00282203

[5] Yang, Z.F. and Qiu, D.H. (2009) Energy Decaying and Blow-Up of Solution for a Kirchhoff Equation with Strong Damping. Journal of Mathematical Research \& Exposition, 29, 707-715.

[6] Kosuke, O. (1997) On Global Existence, Asymptotic Stability and Blowing up of Solutions for Some Degenerate Non-Linear Wave Equations of Kirchhoff Type with a Strong Dissipation. Mathematical Methods in the Applied Sciences, 20, 151-177. https://doi.org/10.1002/(SICI)1099-1476(19970125)20:2<151::AID-MMA851>3.0.C $\underline{\mathrm{O} ; 2-0}$

[7] Lin, X.L. and Li, F.S. (2013) Global Existence and Decay Estimates for Nonlinear Kirchhoff-Type Equation with Boundary Dissipation. Differential Equations \& Applications, 5, 297-317. https://doi.org/10.7153/dea-05-18

[8] Ye, Y.J. (2013) Global Existence and Energy Decay Estimate of Solutions for a Higher-Order Kirchhoff Type Equation with Damping and Source Term. Nonlinear Analysis: Real World Applications, 14, 2059-2067. https://doi.org/10.1016/j.nonrwa.2013.03.001

[9] Li, M.R. and Tsai, L.Y. (2003) Existence and Nonexistence of Global Solutions of Some System of Semilinear Wave Equations. Nonlinear Analysis, 54, 1397-1415. https://doi.org/10.1016/S0362-546X(03)00192-5

[10] Li, F.C. (2004) Global Existence and Blow-Up of Solutions for a Higher-Order Kirchhoff-Type Equation with Nonlinear Dissipation. Applied Mathematics Letters, 17, 1409-1414. https://doi.org/10.1016/j.am1.2003.07.014

[11] Wu, S. and Tsai, L. (2006) Blow-Up of Solutions for Some Nonlinear Wave Equa- 
tions of Kirchhoff Type with Some Dissipation. Nonlinear Analysis. Theory, $\mathrm{Me}$ thods \& Applications, 65, 243-264. https://doi.org/10.1016/j.na.2004.11.023

[12] Gazzola, F. and Squassina, M. (2006) Global Solutions and Finite Time Blow up for Semilinear Wave Equation. Ann. Inst. Henri Poincaré, Anal. Non Linéaire, 23, 185 207.

[13] Lin, G.G. (2011) Nonlinear Evolution Equation. Yunnan University Press, Kunming.

Submit or recommend next manuscript to SCIRP and we will provide best service for you:

Accepting pre-submission inquiries through Email, Facebook, LinkedIn, Twitter, etc. A wide selection of journals (inclusive of 9 subjects, more than 200 journals)

Providing 24-hour high-quality service

User-friendly online submission system

Fair and swift peer-review system

Efficient typesetting and proofreading procedure

Display of the result of downloads and visits, as well as the number of cited articles Maximum dissemination of your research work

Submit your manuscript at: http://papersubmission.scirp.org/

Or contactijmnta@scirp.org 